$1-7-2022$

\title{
Design of SkSP-R Plan for Popular Statistical Distributions
}

Jaffer Hussain

GC University, Lahore, Pakistan, jafferfarooq@hotmail.com

S. Balamurali

Kalasalingam University, Krishnankoil, India, sbmurali@rediffmail.com

Muhammad Aslam

Department of Statistics, Faculty of Science, King Abdulaziz University, Jeddah, Saudi Arabia, aslam_ravian@hotmail.com

Follow this and additional works at: https://digitalcommons.wayne.edu/jmasm

Part of the Applied Statistics Commons, Social and Behavioral Sciences Commons, and the Statistical Theory Commons

\section{Recommended Citation}

Hussain, J., Balamurali, S., \& Aslam, M. (2020). Design of SkSP-R Plan for Popular Statistical Distributions. Journal of Modern Applied Statistical Methods, 19(1), eP2860. https://doi.org/10.22237/jmasm/ 1608552240

This Regular Article is brought to you for free and open access by the Open Access Journals at DigitalCommons@WayneState. It has been accepted for inclusion in Journal of Modern Applied Statistical Methods by an authorized editor of DigitalCommons@WayneState. 


\section{Design of SkSP-R Plan for Popular Statistical Distributions}

\section{Cover Page Footnote}

Muhammad Aslam ORCID: https://orcid.org/0000-0003-0644-1950 


\section{Design of SkSP-R Plan for Popular Statistical Distributions}

\author{
Jaffer Hussain \\ GC University \\ Lahore, Pakistan
}

\author{
S. Balamurali \\ Kalasalingam Univ. \\ Krishnankoil, India
}

\author{
Muhammad Aslam \\ King Abdulaziz Univ. \\ Jeddah, Saudi Arabia
}

The design of a Skip-lot sampling plan of type SkSP-R is presented for time truncated life test for the Weibull, Exponentiated Weibull, and Birnbaum-Saunders lifetime distributions. The plan parameters of the SkSP-R plan under these three distributions are determined through a nonlinear optimization problem. Tables are also constructed for each distribution. The advantages of the proposed plan over the existing sampling schemes are discussed. Application of the proposed plan is explained with the help of an example. The BirnbaumSaunders distribution is economically superior to other two distributions in terms of minimum average sample number.

Keywords: Skip-lot sampling, Life tests, binomial distribution, Weibull distribution, Exponentiated Weibull distribution, Birnbaum-Saunders distribution

\section{Introduction}

A variety of sampling inspection schemes were for life testing of a product. These sampling schemes are applied in many manufacturing industries under various situations. Inspection policies are based on sampling plans and are chosen for various reasons. Sometimes, time is an important constraint so that the sampling scheme chosen for inspection should yield the decision on the disposition of a lot within a short period of time. Sometimes, cost may also be an important factor. In such cases, the sampling schemes opted for inspection should be economical and efficient in minimizing the risks. Taylor (1997) pointed out as "one should follow this approach if you are uncertain of knowing how much sampling or inspection will be conducted on a day-by-day basis" (p. 366). The single sampling plan (SSP) is one of the sampling plans widely used in the industries since it is easier to apply. The double sampling plan can be used when the experimenters are not able to reach

https://doi.org/10.22237/jmasm/1608552240 | Accepted: Jun 29, 2018; Published: Jan 7, 2022. Correspondence: Muhammad Aslam (Department of Statistics, Faculty of Science, https://orcid.org/0000-0003-0644-1950) aslam_ravian@hotmail.com 


\section{HUSSAIN ET AL.}

a decision about the lots based on the first sample. Group sampling plans may be implemented whenever more than one item can be installed on a single tester. Skiplot sampling schemes have been widely applied in industries when the history of quality of the products is excellent. This type of sampling scheme has many applications in chemical and water industries.

The use of skip-lot sampling plan (SkSP) is considered as more efficient than the SSP in terms of minimizing the cost of inspection. These plans consist of two kinds of inspection modes namely, normal inspection mode and skipping inspection mode that makes it useful for practical applications. As mentioned by Hsu (1980), the SkSP has economical advantages in the inspection of final products. The concept of SkSP of type SkSP-1 is the initial version of SkSPs originated by Dodge (1955). The improved version of SkSP-1 plan designated as SkSP-2 was proposed by Perry $(1970,1973)$ by incorporating the concept of reference plan. Aslam et al. (2010) and Balamurali and Subramani (2012) respectively investigated the designing of SkSP-2 plan by using single and double sampling plans as the reference plans. The details and applications of various SkSPs can be seen in Hsu (1980), Parker and Kessler (1981), Carr (1982), Cox (1980), Liebesman (1987), Balamurali et al. (2008), Duffuaa et al. (2009), Aslam et al. (2010), Balamurali and Jun (2011), Aslam et al. (2012a, 2012b, 2013a, 2013b, 2014a, 2014b), Cao and Subramaniam (2013), Balamurali et al. (2014b) and Balamurali and Subramani (2018). Balamurali et al. (2014a) introduced a new type of SkSP designated as SkSP-R plan by incorporating the concept of resampling. Hussain et al. (2014) presented the designing methodology of SkSP-R plan using two points on the operating characteristic (OC) curve approach. In the testing of electronic products, the failure time of the products may follow any non-normal distribution. There was no work on SkSP-R plan using lifetime distributions. Hence the intent is to consider the designing of SkSP-R plan under the three most important life time distributions.

\section{Designing of SkSP-R Plan Under Various Distributions}

In general, while designing the sampling plans for assuring the lifetime of the products under any distribution, the probability that the product will survive up to precise time is determined using the cumulative distribution function (cdf) of the respective distributions. Such probability is also called failure probability. The mean value or percentile value of the distributions is also involved in such calculation regarding to the assurance of mean life or percentile life. In this paper, we design the proposed SkSP-R plan to ensure the mean life of the products. Therefore, we discuss about the failure probability calculation under Weibull, 


\section{SKSP-R PLAN FOR POPULAR STATISTICAL DISTRIBUTIONS}

exponentiated Weibull and Birnbaum-Saunders distributions in the following subsections. In this designing, it is assumed that the scale parameter of each distribution is unknown and shape parameter is known.

\section{Weibull Distribution}

Assume the failure time of the product follows the Weibull distribution with the following cdf.

$$
F(t ; \lambda, w)=1-\exp \left(-(t / \lambda)^{w}\right) \quad t \geq 0, \lambda>0, w>0
$$

where $\lambda$ is the scale parameter and $w$ is the shape parameter of the Weibull distribution. The mean of the Weibull distribution is given as

$$
\mu=(\lambda / w) \Gamma(1 / w)
$$

Express $t_{0}=a \mu_{0}$, where $\mu_{0}$ is the specified average life of the product and $a$ is termination ratio of experiment time. The probability of failure $(p)$ of an item before termination time $t_{0}$ under the Weibull distribution is given as follows

$$
\left.p=1-\exp \left(-\left(t_{0} / \lambda\right)^{w}\right)=1-\exp \left(-a^{w}\left(\frac{\mu}{\mu_{0}}\right)^{-w}\left(\frac{\Gamma(1 / w)}{w}\right)\right)^{w}\right)
$$

Using the above equation, the failure probability may be obtained of an item before the time $t_{0}$ for different combinations of mean ratio, shape parameter and termination ratio.

\section{Exponentiated Weibull Distribution}

The exponentiated Weibull (EW) distribution was developed by Mudholkar and Srivastava (1993). Suppose that the failure time of product follows the EW distribution with following cdf with scale parameter $\lambda$ and shape parameters $w$ and $\gamma$. 


$$
F(t ; \lambda, w, \gamma)=\left[1-\exp \left(-(t / \lambda)^{w}\right)\right]^{\gamma} t \geq 0, \lambda>0, w>0, \gamma>0
$$

The EW distribution is the generalization of several distributions (see Mudholkar and Srivastava, 1993). When $\gamma=1$, EW distribution reduces to Weibull distribution. When $\gamma=1$ and $w=1$, this distribution becomes the exponential distribution. When $w=1$, EW distribution converges to exponentiated exponential distribution. The probability of failure of an item before the termination time $t_{0}$ under the EW distribution is given as

$$
p=F\left(t_{0}\right)=\left[1-\exp \left(-(t / \lambda)^{w}\right)\right]^{\gamma}
$$

The mean of the EW distribution is obtained by the following equation.

$$
\mu=\lambda \gamma \Gamma\left(\frac{1}{w}+1\right) \sum_{i=0}^{\gamma-1}\left(\begin{array}{c}
\gamma-1 \\
i
\end{array}\right)(-1)^{i}(i+1)^{-\left(\frac{1}{w}+1\right)}
$$

The experiment time is expressed as $t_{0}=a \mu_{0}$ and the unknown scale parameter $\lambda$ is represented in terms of mean $\mu$. After the substitution of $t_{0}$ and $\lambda$ in equation (5), the probability of failure of an item before the termination time $t_{0}$ is given as follows.

$$
\left.p=\left\{1-\exp \left[-\left(a \gamma\left(\mu_{0} / \mu\right) \Gamma\left(\frac{1}{w}+1\right) \sum_{i=0}^{\gamma-1}\left(\begin{array}{c}
\gamma-1 \\
i
\end{array}\right)(-1)^{i}(i+1)^{-\left(\frac{1}{w}+1\right)}\right)\right)^{w}\right]\right\}^{\gamma}
$$

Calculate the failure probability of an item using equation (7) when the lifetime of the products follows the EW distribution.

\section{Birnbaum-Saunders Distribution}

Suppose the failure time of the product follows the Birnbaum-Saunders (BS) distribution with cdf given as follows 


$$
F(t ; \lambda, \delta)=\Phi\left[\frac{1}{\delta}\left\{\left(\frac{t}{\lambda}\right)^{1 / 2}-\left(\frac{\lambda}{t}\right)^{1 / 2}\right\}\right] \quad 0<t<\infty, \lambda>0, \delta>0
$$

where $\lambda$ and $\delta$ are scale and shape parameters of BS distribution, respectively. According to Lemonte et al. (2007), the mean life of BS distribution is given by

$$
\mu=\lambda\left(1+\delta^{2} / 2\right)
$$

The probability of failure of an item before $t_{0}$ under the BS distribution is given as

$$
p=\Phi\left[\frac{1}{\delta}\left\{\left(\frac{a\left(1+\delta^{2} / 2\right)}{\mu / \mu_{0}}\right)^{1 / 2}-\left(\frac{\mu / \mu_{0}}{a\left(1+\delta^{2} / 2\right)}\right)^{1 / 2}\right\}\right]
$$

The failure probability of the product under BS distribution can be obtained using the above equation for different values of $a, \delta, \mu / \mu_{0}$.

\section{Designing of SkSP-R Plan based on Time Truncated Life Test}

The operating procedure of SkSP-R plan (see Balamurali et al., 2014a) will be presented. It is an extended version of SkSP-V plan developed by Balamurali and Jun (2011). The following is the operating procedure of the SkSP-R plan under truncated life tests.

Step 1. During normal inspection, select a sample of $n$ items randomly from the submitted lot and conduct the life test on the sample items for the specified duration $t_{0}$. Observe and count the number of sample items which failed before the experiment duration, say, $d$. If $d \leq c$, then accept the lot. Reject the lot if $d>c$.

Step 2. When $i$ consecutive lots are accepted under normal inspection based on time truncated life test, discontinue the normal inspection and switch to the skipping inspection. 


\section{HUSSAIN ET AL.}

Step 3. During skipping inspection, randomly select a fraction $f$ of lots for the inspection and inspect the lots using the procedure as given in Step 1. Continue the skipping inspection until a lot is rejected.

Step 4. If a lot is rejected after $k$ consecutive lots have been accepted, then go for resampling procedure for the successive lot as in Step 5, otherwise switch to normal inspection as in Step 1.

Step 5. Skipping inspection will be continued if a lot is accepted during resampling under truncated life test. The resampling procedure is followed on non-acceptance lot for $m$ times using the procedure as in Step 1. The lot is accepted under resampling procedure if it has been accepted on $(m-1)^{\text {th }}$ resubmission. Otherwise, reject the lot.

Step 6. Switch back to normal inspection as in Step 1 if a lot is rejected under resampling procedure.

Step 7. The failed items contained in the rejected lots are replaced with nonfailed items.

The proposed SkSP-R plan is completely specified by six parameters which are given below.

1. Sample size of reference plan, say $n$.

2. Acceptance number of reference plan, say $c$.

3. The fraction of lots inspected during skipping sampling mode, say $f$ $(0<f<1)$

4. The clearance number for the normal inspection mode, say $i$

5. The clearance number of skipping inspection mode, say $k$

6. The number of times, resubmission of lots for inspection is allowed, $m$

The proposed plan is designated as $\operatorname{SkSP}-\mathrm{R}(n, c, f, i, k, m)$. According to Balamurali et al. (2014a), the OC function of the SkSP-R plan is given as 


$$
P_{a}(p)=\frac{f P+(1-f) P^{i}+f P^{k}\left(P^{i}-P\right)\left(1-Q^{m}\right)}{f\left(1-P^{i}\right)\left[1-P^{k}\left(1-Q^{m}\right)\right]+P^{i}\left(1+f Q P^{k}\right)}
$$

Similarly, the average sample number (ASN) function of the SkSP-R plan (see Balamurali et al., 2014a) is given as

$$
A S N(p)=\frac{n f+n f Q P^{i+k}-n f P^{k}\left(1-P^{i}\right)\left(1-Q^{m}\right)}{f\left(1-P^{i}\right)\left[1-P^{k}\left(1-Q^{m}\right)\right]+P^{i}\left(1+f Q P^{k}\right)}
$$

where $P$ is the probability of acceptance of a lot based on reference plan SSP and $Q=1-P$. Now, $P$ and $Q$ are determined as

$$
P=\sum_{i=0}^{c}\left(\begin{array}{c}
n \\
i
\end{array}\right) p^{i}(1-p)^{n-i} \text { and } Q=1-P
$$

where $p$ is the failure probability of an item. The sampling plans are usually designed to minimize the producer's risk $(\alpha)$ and consumer's risk $(\beta)$. The producer always wants that the acceptance probability of a good lot should be larger than $(1-\alpha)$ at acceptable quality level (AQL) and the consumer always demands that acceptance probability of a bad lot should be smaller than $\beta$ at limiting quality level (LQL). The AQL and LQL are respectively denoted by $p_{1}$ and $p_{2}$. The sampling plans are designed so that the OC curve passes through the two points $\left(p_{1}, 1-\alpha\right)$ and $\left(p_{2}, \beta\right)$. In order to determine the optimal parameters of the SkSP-R plan, we use the following nonlinear optimization problem, in which the objective function is to minimize ASN function at LQL and the corresponding constraints are the lot acceptance probabilities at AQL and LQL respectively.

Minimize

$$
A S N\left(p_{2}\right)=\frac{n f+n f Q_{2} P_{2}^{i+k}-n f P_{2}^{k}\left(1-P_{2}^{i}\right)\left(1-Q_{2}{ }^{m}\right)}{f\left(1-P_{2}^{i}\right)\left[1-P_{2}^{k}\left(1-Q_{2}{ }^{m}\right)\right]+P_{2}^{i}\left(1+f Q_{2} P_{2}^{k}\right)}
$$

Subject to 


$$
\begin{gathered}
P_{a}\left(p_{1}\right)=\frac{f P_{1}+(1-f) P_{1}^{i}+f P_{1}^{k}\left(P_{1}^{i}-P_{1}\right)\left(1-Q_{1}^{m}\right)}{f\left(1-P_{1}^{i}\right)\left[1-P_{1}^{k}\left(1-Q_{1}^{m}\right)\right]+P_{1}^{i}\left(1+f Q_{1} P_{1}^{k}\right)} \geq 1-\alpha \\
P_{a}\left(p_{1}\right)=\frac{f P_{2}+(1-f) P_{2}^{i}+f P_{2}^{k}\left(P_{2}^{i}-P_{2}\right)\left(1-Q_{2}^{m}\right)}{f\left(1-P_{2}^{i}\right)\left[1-P_{2}^{k}\left(1-Q_{2}{ }^{m}\right)\right]+P_{2}^{i}\left(1+f Q_{2} P_{2}^{k}\right)} \leq \beta \\
n>1, c \geq 0, f>0, i>1, k \geq 1, m>1
\end{gathered}
$$

where $P_{1}$ and $P_{2}$ are evaluated at $\mathrm{AQL}$ and LQL respectively, and $Q_{1}=1-P_{1}$, $Q_{2}=1-P_{2}$. In this paper, we consider the failure probabilities respective to the mean ratios $\mu / \mu_{0}=2,4,6,8,10,12$ are as $\mathrm{AQL}$ and the failure probability at $\mu / \mu_{0}=1$ is assumed as LQL. Determine the optimal parameters so that the fixed value of producer's risk $\alpha=0.05$, four different values of consumer's risk $\beta=0.25$, $0.10,0.05,0.10$ are satisfied with minimum ASN and two cases of experiment termination ratio $a=0.5$ and $a=1.0$ are considered. The optimal plan parameters of the proposed SkSP-R plan under above mentioned three distributions are determined by solving the non-linear optimization problem as given in (14a) through (14c). Determine the optimal parameters for fixed value of $m=2$, since Govindaraju and Ganesalingam (1997) has pointed out that the optimum value of $m$ is 2 . For determining the optimal parameters under Weibull distribution, three values of shape parameters namely $w=1,2$ and 3 are considered and the optimal parameters are reported in Tables 1-3.

From these tables, it is observed that the sample size as well as the ASN decreases if there is an increment in mean ratios and $w=1$. But there is no trend in sample size and ASN for other two shape parameters. For fixed values of $\beta, w, \mu / \mu_{0}$, the sample size and ASN decrease when $a$ increases from 0.5 to 1.0. Similarly, the sample size and ASN increase if there is decrement in consumer's risk and $w=1,2$. It is noticed from Tables 1 and 2 that the maximum value of $i=2$ and $k=1$. 


\section{SKSP-R PLAN FOR POPULAR STATISTICAL DISTRIBUTIONS}

Table 1. Optimal parameters of SkSP-R Plan with $m=2$ under the Weibull Distribution with shape parameter $w=1$

\begin{tabular}{|c|c|c|c|c|c|c|c|}
\hline \multirow{2}{*}{$\beta$} & \multirow{2}{*}{$\mu / \mu_{0}$} & \multicolumn{3}{|c|}{$a=0.5$} & \multicolumn{3}{|c|}{$a=1.0$} \\
\hline & & $n, c, i, f, k$ & $P_{a}\left(p_{1}\right)$ & $\operatorname{ASN}\left(p_{2}\right)$ & $n, c, i, f, k$ & $P_{a}\left(p_{1}\right)$ & $\operatorname{ASN}\left(p_{2}\right)$ \\
\hline \multirow{6}{*}{0.25} & 2 & $15,3,2,0.05,1$ & 0.95484 & 12.607 & $12,5,2,0.10,1$ & 0.95493 & 10.846 \\
\hline & 4 & $8,1,2,0.10,1$ & 0.97105 & 7.153 & $4,1,2,0.15,1$ & 0.96351 & 3.565 \\
\hline & 6 & $5,0,2,0.05,1$ & 0.97343 & 4.426 & $3,0,2,0.05,1$ & 0.96394 & 2.864 \\
\hline & 8 & $4,0,2,0.15,1$ & 0.96192 & 3.613 & $2,0,2,0.15,1$ & 0.96192 & 1.806 \\
\hline & 10 & $\uparrow$ & 0.97041 & $\uparrow$ & $\uparrow$ & 0.97041 & $\uparrow$ \\
\hline & 12 & $\uparrow$ & 0.97566 & $\uparrow$ & $\uparrow$ & 0.97566 & $\uparrow$ \\
\hline \multirow{6}{*}{0.1} & 2 & $24,5,2,0.05,1$ & 0.95140 & 23.077 & $18,7,2,0.05,1$ & 0.95936 & 17.674 \\
\hline & 4 & $10,1,2,0.05,1$ & 0.97473 & 9.537 & $5,1,2,0.10,1$ & 0.95763 & 4.817 \\
\hline & 6 & $6,0,2,0.05,1$ & 0.96394 & 5.729 & $3,0,2,0.05,1$ & 0.96394 & 2.864 \\
\hline & 8 & $\uparrow$ & 0.97740 & $\uparrow$ & $\uparrow$ & 0.97740 & $\uparrow$ \\
\hline & 10 & $\uparrow$ & 0.98347 & $\uparrow$ & $\uparrow$ & 0.98347 & $\uparrow$ \\
\hline & 12 & $\uparrow$ & 0.98688 & $\uparrow$ & $\uparrow$ & 0.98688 & $\uparrow$ \\
\hline \multirow{6}{*}{0.05} & 2 & $32,7,2,0.05,1$ & 0.95941 & 31.482 & $18,7,2,0.05,1$ & 0.95936 & 17.674 \\
\hline & 4 & $12,1,2,0.05,1$ & 0.95781 & 11.893 & $6,1,2,0.05,1$ & 0.96327 & 5.911 \\
\hline & 6 & $7,0,2,0.05,1$ & 0.95208 & 6.881 & $\uparrow$ & 0.98599 & $\uparrow$ \\
\hline & 8 & $\uparrow$ & 0.97126 & $\uparrow$ & $4,0,2,0.05,1$ & 0.96394 & 3.975 \\
\hline & 10 & $\uparrow$ & 0.97957 & $\uparrow$ & $\uparrow$ & 0.97507 & $\uparrow$ \\
\hline & 12 & $\uparrow$ & 0.98407 & $\uparrow$ & $\uparrow$ & 0.98093 & $\uparrow$ \\
\hline \multirow{6}{*}{0.01} & 2 & * & * & * & * & * & * \\
\hline & 4 & $19,2,2,0.05,1$ & 0.96473 & 18.985 & $10,2,2,0.05,1$ & 0.96532 & 9.991 \\
\hline & 6 & $14,1,2,0.10,1$ & 0.95689 & 13.989 & $8,1,2,0.05,1$ & 0.97132 & 7.996 \\
\hline & 8 & $\uparrow$ & 0.97641 & $\uparrow$ & $\uparrow$ & 0.98525 & $\uparrow$ \\
\hline & 10 & $10,0,2,0.05,1$ & 0.96394 & 9.991 & $5,0,2,0.05,1$ & 0.96394 & 4.996 \\
\hline & 12 & $\uparrow$ & 0.97342 & $\uparrow$ & $\uparrow$ & 0.97343 & $\uparrow$ \\
\hline
\end{tabular}

*: Plan doesn't exist; $\uparrow:$ Use the plan above. 
HUSSAIN ET AL.

Table 2. Optimal parameters of SkSP-R Plan with $m=2$ under the Weibull Distribution with shape parameter $w=2$

\begin{tabular}{|c|c|c|c|c|c|c|c|}
\hline \multirow{2}{*}{$\beta$} & \multirow{2}{*}{$\mu / \mu_{0}$} & \multicolumn{3}{|c|}{$a=0.5$} & \multicolumn{3}{|c|}{$a=1.0$} \\
\hline & & $n, c, i, f, k$ & $P_{a}\left(p_{1}\right)$ & $\operatorname{ASN}\left(p_{2}\right)$ & $n, c, i, f, k$ & $P_{a}\left(p_{1}\right)$ & $\operatorname{ASN}\left(p_{2}\right)$ \\
\hline \multirow{6}{*}{0.25} & 2 & $12,0,2,0.05,1$ & 0.95113 & 10.228 & $3,0,2,0.05,1$ & 0.95113 & 2.557 \\
\hline & 4 & $8,0,2,0.5,1$ & 0.95427 & 7.646 & $2,0,2,0.5,1$ & 0.95427 & 1.912 \\
\hline & 6 & $\uparrow$ & 0.97899 & $\uparrow$ & $\uparrow$ & 0.97899 & $\uparrow$ \\
\hline & 8 & $\uparrow$ & 0.98800 & $\uparrow$ & $\uparrow$ & 0.98800 & $\uparrow$ \\
\hline & 10 & $8,0,2,0.65,1$ & 0.98996 & 7.806 & $2,0,2,0.65,1$ & 0.98996 & 1.951 \\
\hline & 12 & $9,0,2,0.85,1$ & 0.98973 & 8.952 & $2,0,2,0.65,1$ & 0.98955 & 2.985 \\
\hline \multirow{6}{*}{0.1} & 2 & $24,1,2,0.10,1$ & 0.95419 & 23.344 & $7,1,2,0.05,1$ & 0.96978 & 6.810 \\
\hline & 4 & $13,0,2,0.20,1$ & 0.96926 & 12.689 & $4,0,2,0.10,1$ & 0.98042 & 3.934 \\
\hline & 6 & $12,0,2,0.65,1$ & 0.96005 & 11.941 & $3,0,2,0.65,1$ & 0.96005 & 2.985 \\
\hline & 8 & $\uparrow$ & 0.97696 & $\uparrow$ & $\uparrow$ & 0.97696 & $\uparrow$ \\
\hline & 10 & $\uparrow$ & 0.98506 & $\uparrow$ & $\uparrow$ & 0.98506 & $\uparrow$ \\
\hline & 12 & $\uparrow$ & 0.98955 & $\uparrow$ & $\uparrow$ & 0.98955 & $\uparrow$ \\
\hline \multirow{6}{*}{0.05} & 2 & $28,1,2,0.05,1$ & 0.96449 & 27.561 & $8,1,2,0.05,1$ & 0.95495 & 7.941 \\
\hline & 4 & $16,0,2,0.25,1$ & 0.95276 & 15.911 & $4,0,2,0.25,1$ & 0.95276 & 3.978 \\
\hline & 6 & $\uparrow$ & 0.97909 & $\uparrow$ & $\uparrow$ & 0.97909 & $\uparrow$ \\
\hline & 8 & $\uparrow$ & 0.98808 & $\uparrow$ & $\uparrow$ & 0.98808 & $\uparrow$ \\
\hline & 10 & $16,0,2,0.35,1$ & 0.98926 & 15.945 & $4,0,2,0.35,1$ & 0.98926 & 3.986 \\
\hline & 12 & $16,0,2,0.50,1$ & 0.98931 & 15.970 & $4,0,2,0.50,1$ & 0.98931 & 3.993 \\
\hline \multirow{6}{*}{0.01} & 2 & $45,2,2,0.05,1$ & 0.96915 & 44.941 & $13,2,2,0.05,1$ & 0.95869 & 12.995 \\
\hline & 4 & $24,0,2,0.10,1$ & 0.96839 & 23.983 & $6,0,2,0.10,1$ & 0.96839 & 5.996 \\
\hline & 6 & $\uparrow$ & 0.98725 & $\uparrow$ & $\uparrow$ & 0.98725 & $\uparrow$ \\
\hline & 8 & $24,0,2,0.15,1$ & 0.98929 & 23.989 & $6,0,2,0.15,1$ & 0.98929 & 5.997 \\
\hline & 10 & $24,0,2,0.25,1$ & 0.98855 & 23.994 & $6,0,2,0.25,1$ & 0.98855 & 5.998 \\
\hline & 12 & $24,0,2,0.35,1$ & 0.98882 & 23.996 & $6,0,2,0.35,1$ & 0.98882 & 5.999 \\
\hline
\end{tabular}

$\uparrow:$ Use the plan above. 


\section{SKSP-R PLAN FOR POPULAR STATISTICAL DISTRIBUTIONS}

Table 3. Optimal parameters of SkSP-R Plan with $m=2$ under the Weibull Distribution with shape parameter $w=3$

\begin{tabular}{|c|c|c|c|c|c|c|c|}
\hline \multirow{2}{*}{$\beta$} & \multirow{2}{*}{$\mu / \mu_{0}$} & \multicolumn{3}{|c|}{$a=0.5$} & \multicolumn{3}{|c|}{$a=1.0$} \\
\hline & & $n, c, i, f, k$ & $P_{a}\left(p_{1}\right)$ & $\operatorname{ASN}\left(p_{2}\right)$ & $n, c, i, f, k$ & $P_{a}\left(p_{1}\right)$ & $\operatorname{ASN}\left(p_{2}\right)$ \\
\hline \multirow{6}{*}{0.25} & 2 & $21,0,2,0.20,1$ & 0.95373 & 19.106 & $3,0,2,0.10,1$ & 0.97205 & 2.658 \\
\hline & 4 & $16,0,3,0.6,1$ & 0.98695 & 15.837 & $2,0,3,0.6,1$ & 0.98695 & 1.979 \\
\hline & 6 & $29,0,3,0.85,2$ & 0.98999 & 28.998 & $4,0,2,0.80,1$ & 0.98962 & 3.996 \\
\hline & 8 & $69,0,2,0.85,1$ & 0.98996 & 68.999 & $9,0,2,0.85,1$ & 0.98953 & 8.999 \\
\hline & 10 & $135,0,2,0.85,1$ & 0.98995 & 135.000 & $17,0,2,0.85,1$ & 0.98987 & 17.000 \\
\hline & 12 & $232,0,3,0.85,2$ & 0.99000 & 232.000 & $29,0,3,0.85,2$ & 0.98999 & 29.000 \\
\hline \multirow{6}{*}{0.1} & 2 & $31,0,2,0.10,1$ & 0.96104 & 29.912 & $4,0,2,0.10,1$ & 0.95928 & 3.882 \\
\hline & 4 & $26,0,3,0.45,1$ & 0.98420 & 25.969 & $4,0,2,0.25,1$ & 0.98918 & 3.960 \\
\hline & 6 & $29,0,3,0.85,2$ & 0.98999 & 28.998 & $4,0,2,0.80,1$ & 0.98962 & 3.997 \\
\hline & 8 & $69,0,2,0.85,1$ & 0.98996 & 68.999 & $9,0,2,0.85,1$ & 0.98953 & 8.999 \\
\hline & 10 & $135,0,2,0.85,1$ & 0.98995 & 135.000 & $17,0,2,0.85,1$ & 0.98987 & 17.000 \\
\hline & 12 & $232,0,3,0.85,2$ & 0.99000 & 232.000 & $29,0,3,0.85,2$ & 0.98999 & 29.000 \\
\hline \multirow{6}{*}{0.05} & 2 & $39,0,2,0.05,1$ & 0.97164 & 38.296 & $5,0,2,0.05,1$ & 0.97044 & 4.924 \\
\hline & 4 & $34,0,2,0.60,1$ & 0.97289 & 33.945 & $5,0,2,0.20,1$ & 0.98920 & 4.983 \\
\hline & 6 & $34,0,2,0.75,1$ & 0.98967 & 33.973 & $5,0,2,0.65,1$ & 0.98948 & 4.998 \\
\hline & 8 & $69,0,2,0.85,1$ & 0.98996 & 68.999 & $9,0,2,0.85,1$ & 0.98953 & 8.999 \\
\hline & 10 & $135,0,2,0.85,1$ & 0.98994 & 135.000 & $17,0,2,0.85,1$ & 0.98987 & 17.000 \\
\hline & 12 & $232,0,3,0.85,2$ & 0.99000 & 232.000 & $29,0,3,0.85,2$ & 0.98999 & 29.000 \\
\hline \multirow{6}{*}{0.01} & 2 & $76,1,2,0.15,1$ & 0.96537 & 75.963 & $10,1,2,0.10,1$ & 0.97636 & 9.992 \\
\hline & 4 & $52,0,2,0.30,1$ & 0.97921 & 51.988 & $7,0,2,0.15,1$ & 0.98869 & 6.998 \\
\hline & 6 & $52,0,2,0.50,1$ & 0.98949 & 51.995 & $7,0,2,0.45,1$ & 0.98983 & 6.999 \\
\hline & 8 & $69,0,2,0.85,1$ & 0.98996 & 68.999 & $9,0,2,0.85,1$ & 0.98953 & 8.999 \\
\hline & 10 & $135,0,2,0.85,1$ & 0.98995 & 135.000 & $17,0,2,0.85,1$ & 0.98987 & 17.000 \\
\hline & 12 & $232,0,3,0.85,2$ & 0.99000 & 232.000 & $29,0,3,0.85,2$ & 0.98999 & 29.000 \\
\hline
\end{tabular}

Under EW distribution, the optimal parameters are determined for two different combinations of shape parameters such as $w=1, \gamma=0.5$ and $w=3$, $\gamma=0.5$. The optimal parameters of the proposed plan obtained under EW distribution are presented in Tables 4 and 5. It is observed from Table 4 that the optimal plan does not exist at $\mu / \mu_{0}=2$ when $w=1, \gamma=0.5$. The decreasing trend in sample size and ASN can be observed if there is increment in either mean ratios or in experiment termination ratio. But the sample size and ASN increase when consumer's risk decreases. The same trend is also observed from Table 5. In 


\section{HUSSAIN ET AL.}

addition, if one of the shape parameters namely $w$ increases, then the sample size and ASN are also increased.

Table 4. Optimal parameters of SkSP-R Plan with $m=2$ under the EW Distribution with shape parameter $w=1$ and $y=0.5$

\begin{tabular}{|c|c|c|c|c|c|c|c|}
\hline \multirow{2}{*}{$\beta$} & \multirow{2}{*}{$\mu / \mu_{0}$} & \multicolumn{3}{|c|}{$a=0.5$} & \multicolumn{3}{|c|}{$a=1.0$} \\
\hline & & $n, c, i, f, k$ & $P_{a}\left(p_{1}\right)$ & $\operatorname{ASN}\left(p_{2}\right)$ & $n, c, i, f, k$ & $P_{a}\left(p_{1}\right)$ & $\operatorname{ASN}\left(p_{2}\right)$ \\
\hline \multirow{6}{*}{0.25} & 2 & * & * & * & * & * & * \\
\hline & 4 & $13,3,2,0.05,1$ & 0.96193 & 11.858 & $9,3,2,0.05,1$ & 0.96797 & 8.186 \\
\hline & 6 & $7,1,2,0.05,1$ & 0.95565 & 6.156 & $5,1,2,0.05,1$ & 0.95373 & 4.596 \\
\hline & 8 & $\uparrow$ & 0.97119 & $\uparrow$ & $4,1,2,0.15,1$ & 0.95355 & 3.536 \\
\hline & 10 & $6,1,2,0.15,1$ & 0.95735 & 5.384 & $\uparrow$ & 0.96364 & $\uparrow$ \\
\hline & 12 & $\uparrow$ & 0.96475 & $\uparrow$ & $\uparrow$ & 0.96988 & $\uparrow$ \\
\hline \multirow{6}{*}{0.1} & 2 & * & * & * & * & * & * \\
\hline & 4 & $17,4,2,0.05,1$ & 0.95998 & 16.440 & $12,4,2,0.05,1$ & 0.96347 & 11.686 \\
\hline & 6 & $11,2,2,0.05,1$ & 0.96481 & 10.502 & $8,2,2,0.05,1$ & 0.96159 & 7.818 \\
\hline & 8 & $8,1,2,0.05,1$ & 0.95728 & 7.633 & $7,2,2,0.15,1$ & 0.95851 & 6.797 \\
\hline & 10 & $\uparrow$ & 0.96937 & $\uparrow$ & $6,1,2,0.05,1$ & 0.96346 & 5.901 \\
\hline & 12 & $\uparrow$ & 0.97609 & $\uparrow$ & $5,1,2,0.15,1$ & 0.95039 & 4.872 \\
\hline \multirow{6}{*}{0.05} & 2 & * & * & * & * & * & * \\
\hline & 4 & $21,5,2,0.05,1$ & 0.95862 & 20.739 & $15,5,2,0.05,1$ & 0.95978 & 14.886 \\
\hline & 6 & $12,2,2,0.05,1$ & 0.95010 & 11.784 & $11,3,2,0.05,1$ & 0.96666 & 10.928 \\
\hline & 8 & $\uparrow$ & 0.97136 & $\uparrow$ & $9,2,2,0.05,1$ & 0.96497 & 8.954 \\
\hline & 10 & $9,1,2,0.05,1$ & 0.95663 & 8.853 & $6,1,2,0.05,1$ & 0.96346 & 5.901 \\
\hline & 12 & $\uparrow$ & 0.96700 & $\uparrow$ & $\uparrow$ & 0.97204 & $\uparrow$ \\
\hline \multirow{6}{*}{0.01} & 2 & * & * & * & * & * & * \\
\hline & 4 & $33,8,2,0.05,1$ & 0.95632 & 32.976 & $21,7,2,0.05,1$ & 0.95385 & 20.986 \\
\hline & 6 & $21,4,2,0.05,1$ & 0.95709 & 20.976 & $15,4,2,0.05,1$ & 0.95664 & 14.993 \\
\hline & 8 & $18,3,2,0.05,1$ & 0.96449 & 17.980 & $13,3,2,0.05,1$ & 0.96193 & 12.995 \\
\hline & 10 & $15,2,2,0.05,1$ & 0.95618 & 14.986 & $10,2,2,0.05,1$ & 0.96554 & 9.989 \\
\hline & 12 & $\uparrow$ & 0.96895 & $\uparrow$ & $\uparrow$ & 0.97527 & $\uparrow$ \\
\hline
\end{tabular}

*: Plan doesn't exist; $\uparrow:$ Use the plan above. 


\section{SKSP-R PLAN FOR POPULAR STATISTICAL DISTRIBUTIONS}

Table 5. Optimal parameters of SkSP-R Plan with $m=2$ under the EW Distribution with shape parameters $w=3$ and $y=0.5$

\begin{tabular}{|c|c|c|c|c|c|c|c|}
\hline \multirow{2}{*}{$\beta$} & \multirow{2}{*}{$\mu / \mu_{0}$} & \multicolumn{3}{|c|}{$a=0.5$} & \multicolumn{3}{|c|}{$a=1.0$} \\
\hline & & $n, c, i, f, k$ & $P_{a}\left(p_{1}\right)$ & $\operatorname{ASN}\left(p_{2}\right)$ & $n, c, i, f, k$ & $P_{a}\left(p_{1}\right)$ & $\operatorname{ASN}\left(p_{2}\right)$ \\
\hline \multirow{6}{*}{0.25} & 2 & $36,1,2,0.05,1$ & 0.96461 & 30.585 & $12,1,2,0.05,1$ & 0.96947 & 10.232 \\
\hline & 4 & $18,0,2,0.15,1$ & 0.96387 & 16.260 & $6,0,2,0.15,1$ & 0.96577 & 5.491 \\
\hline & 6 & $15,0,2,0.35,1$ & 0.96434 & 14.016 & $5,0,2,0.35,1$ & 0.96611 & 4.708 \\
\hline & 8 & $13,0,2,0.85,1$ & 0.95191 & 12.862 & $\uparrow$ & 0.97776 & $\uparrow$ \\
\hline & 10 & $\uparrow$ & 0.96505 & $\uparrow$ & $\uparrow$ & 0.98396 & $\uparrow$ \\
\hline & 12 & $\uparrow$ & 0.97307 & $\uparrow$ & $\uparrow$ & 0.98773 & $\uparrow$ \\
\hline \multirow{6}{*}{0.1} & 2 & $58,2,2,0.05,1$ & 0.96891 & 55.493 & $20,2,2,0.05,1$ & 0.97153 & 19.356 \\
\hline & 4 & $27,0,2,0.05,1$ & 0.97886 & 25.782 & $8,0,2,0.15,1$ & 0.95170 & 7.821 \\
\hline & 6 & $23,0,2,0.25,1$ & 0.96045 & 22.587 & $\uparrow$ & 0.97606 & $\uparrow$ \\
\hline & 8 & $22,0,2,0.35,1$ & 0.96600 & 21.693 & $7,0,2,0.45,1$ & 0.96078 & 6.931 \\
\hline & 10 & $21,0,2,0.75,1$ & 0.95121 & 20.933 & $\uparrow$ & 0.97161 & $\uparrow$ \\
\hline & 12 & $\uparrow$ & 0.96227 & $\uparrow$ & $\uparrow$ & 0.97822 & $\uparrow$ \\
\hline \multirow{6}{*}{0.05} & 2 & $64,2,2,0.05,1$ & 0.95574 & 62.948 & $22,2,2,0.05,1$ & 0.95978 & 21.744 \\
\hline & 4 & $32,0,2,0.05,1$ & 0.97260 & 31.512 & $10,0,2,0.05,1$ & 0.97696 & 9.812 \\
\hline & 6 & $29,0,2,0.15,1$ & 0.96896 & 28.741 & $\uparrow$ & 0.98960 & $\uparrow$ \\
\hline & 8 & $28,0,2,0.35,1$ & 0.95697 & 27.897 & $9,0,2,0.35,1$ & 0.96066 & 8.966 \\
\hline & 10 & $\uparrow$ & 0.96905 & $\uparrow$ & $\uparrow$ & 0.97165 & $\uparrow$ \\
\hline & 12 & $\uparrow$ & 0.97622 & $\uparrow$ & $\uparrow$ & 0.97827 & $\uparrow$ \\
\hline \multirow{6}{*}{0.01} & 2 & $109,4,2,0.05,1$ & 0.96592 & 108.861 & $32,3,2,0.05,1$ & 0.95303 & 31.969 \\
\hline & 4 & $43,0,2,0.05,1$ & 0.95404 & 42.942 & $14,0,2,0.05,1$ & 0.95971 & 13.983 \\
\hline & 6 & $42,0,2,0.15,1$ & 0.95199 & 41.979 & $\uparrow$ & 0.98440 & $\uparrow$ \\
\hline & 8 & $\uparrow$ & 0.97100 & $\uparrow$ & $\uparrow$ & 0.99069 & $\uparrow$ \\
\hline & 10 & $42,0,2,0.15,2$ & 0.97908 & $\uparrow$ & $\uparrow$ & 0.99349 & $\uparrow$ \\
\hline & 12 & $\uparrow$ & 0.98428 & $\uparrow$ & $\uparrow$ & 0.99508 & $\uparrow$ \\
\hline
\end{tabular}

$\uparrow:$ Use the plan above.

Tables 6-8 are provided for the selection of optimal parameters of the proposed plan for assuring mean life of the products under BS distribution. In order to determine the optimal parameters, the shape parameter values are considered as $\delta=1,2,3$. From Table 6 , it is understood the sample size and ASN decrease if either mean ratio increases or experiment termination ratio increases. The value of $f$ either increases or remaining constant when mean ratio increases. Although the same trend on sample size and ASN can be noticed from Tables 7 and 8 , no certain change is observed in $f$ values. When shape parameter $\delta=2$ and $\mu / \mu_{0}=2$, the 


\section{HUSSAIN ET AL.}

optimal plan does not exist. Similarly, when shape parameter $\delta=3$, the optimal plan does not exist even for the mean ratio 6 at some of the cases.

Table 6. Optimal parameters of SkSP-R Plan with $m=2$ under the BS Distribution with shape parameter $\delta=1$

\begin{tabular}{|c|c|c|c|c|c|c|c|}
\hline \multirow{2}{*}{$\beta$} & \multirow{2}{*}{$\mu / \mu_{0}$} & \multicolumn{3}{|c|}{$a=0.5$} & \multicolumn{3}{|c|}{$a=1.0$} \\
\hline & & $n, c, i, f, k$ & $P_{a}\left(p_{1}\right)$ & $\operatorname{ASN}\left(p_{2}\right)$ & $n, c, i, f, k$ & $P_{a}\left(p_{1}\right)$ & $\overline{A S N}\left(p_{2}\right)$ \\
\hline \multirow{6}{*}{0.25} & 2 & $9,1,2,0.05,1$ & 0.95951 & 7.965 & $8,3,2,0.05,1$ & 0.96769 & 6.777 \\
\hline & 4 & $4,0,2,0.15,1$ & 0.98217 & 3.578 & $3,0,2,0.05,1$ & 0.96385 & 2.912 \\
\hline & 6 & $3,0,2,0.75,1$ & 0.98531 & 2.943 & $2,0,2,0.15,1$ & 0.97994 & 1.854 \\
\hline & 8 & $\uparrow$ & 0.99655 & $\uparrow$ & $\uparrow$ & 0.99103 & $\uparrow$ \\
\hline & 10 & $\uparrow$ & 0.99918 & $\uparrow$ & $\uparrow$ & 0.99581 & $\uparrow$ \\
\hline & 12 & $\uparrow$ & 0.99980 & $\uparrow$ & $\uparrow$ & 0.99801 & $\uparrow$ \\
\hline \multirow{6}{*}{0.1} & 2 & $14,2,2,0.05,1$ & 0.96901 & 13.381 & $11,4,2,0.05,1$ & 0.95540 & 10.608 \\
\hline & 4 & $6,0,2,0.10,1$ & 0.98171 & 5.849 & $3,0,2,0.05,1$ & 0.96385 & 2.912 \\
\hline & 6 & $5,0,2,0.35,1$ & 0.98859 & 4.930 & $\uparrow$ & 0.98947 & $\uparrow$ \\
\hline & 8 & $\uparrow$ & 0.99732 & $\uparrow$ & $\uparrow$ & 0.99550 & $\uparrow$ \\
\hline & 10 & $\uparrow$ & 0.99936 & $\uparrow$ & $\uparrow$ & 0.99791 & $\uparrow$ \\
\hline & 12 & $\uparrow$ & 0.99985 & $\uparrow$ & $\uparrow$ & 0.99901 & $\uparrow$ \\
\hline \multirow{6}{*}{0.05} & 2 & $19,3,2,0.05,1$ & 0.97460 & 18.678 & $16,6,2,0.05,1$ & 0.95587 & 15.890 \\
\hline & 4 & $7,0,2,0.10,1$ & 0.97829 & 6.933 & $6,1,2,0.05,1$ & 0.98595 & 5.955 \\
\hline & 6 & $7,0,2,0.15,1$ & 0.99314 & 6.958 & $3,0,2,0.15,1$ & 0.96920 & 2.973 \\
\hline & 8 & $\uparrow$ & 0.99839 & $\uparrow$ & $\uparrow$ & 0.98662 & $\uparrow$ \\
\hline & 10 & $\uparrow$ & 0.99962 & $\uparrow$ & $\uparrow$ & 0.99375 & $\uparrow$ \\
\hline & 12 & $\uparrow$ & 0.99991 & $\uparrow$ & $\uparrow$ & 0.99703 & $\uparrow$ \\
\hline \multirow{6}{*}{0.01} & 2 & $27,4,2,0.05,1$ & 0.96197 & 26.976 & $21,8,2,0.05,1$ & 0.95660 & 20.971 \\
\hline & 4 & $10,0,2,0.05,1$ & 0.98291 & 9.989 & $7,1,2,0.05,1$ & 0.97975 & 6.992 \\
\hline & 6 & $\uparrow$ & 0.99673 & $\uparrow$ & $5,0,2,0.05,1$ & 0.97993 & 4.998 \\
\hline & 8 & $\uparrow$ & 0.99924 & $\uparrow$ & $\uparrow$ & 0.99213 & $\uparrow$ \\
\hline & 10 & $\uparrow$ & 0.99982 & $\uparrow$ & $\uparrow$ & 0.99650 & $\uparrow$ \\
\hline & 12 & $\uparrow$ & 0.99996 & $\uparrow$ & $\uparrow$ & 0.99835 & $\uparrow$ \\
\hline
\end{tabular}

$\uparrow:$ Use the plan above. 


\section{SKSP-R PLAN FOR POPULAR STATISTICAL DISTRIBUTIONS}

Table 7. Optimal parameters of SkSP-R Plan with $m=2$ under the BS Distribution with shape parameter $\delta=2$

\begin{tabular}{|c|c|c|c|c|c|c|c|}
\hline \multirow{2}{*}{$\beta$} & \multirow{2}{*}{$\mu / \mu_{0}$} & \multicolumn{3}{|c|}{$a=0.5$} & \multicolumn{3}{|c|}{$a=1.0$} \\
\hline & & $n, c, i, f, k$ & $P_{a}\left(p_{1}\right)$ & $\operatorname{ASN}\left(p_{2}\right)$ & $n, c, i, f, k$ & $P_{a}\left(p_{1}\right)$ & $\operatorname{ASN}\left(p_{2}\right)$ \\
\hline \multirow{6}{*}{0.25} & 2 & * & * & * & * & * & * \\
\hline & 4 & $9,3,2,0.10,1$ & 0.96319 & 7.908 & $9,4,2,0.05,1$ & 0.97009 & 8.056 \\
\hline & 6 & $5,1,2,0.10,1$ & 0.95485 & 4.560 & $5,2,2,0.15,1$ & 0.95392 & 4.485 \\
\hline & 8 & $3,0,2,0.05,1$ & 0.95358 & 2.717 & $4,1,2,0.05,1$ & 0.97082 & 3.651 \\
\hline & 10 & $\uparrow$ & 0.97105 & $\uparrow$ & $\uparrow$ & 0.98119 & $\uparrow$ \\
\hline & 12 & $\uparrow$ & 0.98017 & $\uparrow$ & $2,0,2,0.05,1$ & 0.96213 & 1.784 \\
\hline \multirow{6}{*}{0.1} & 2 & * & * & * & * & * & * \\
\hline & 4 & $11,3,2,0.05,1$ & 0.95125 & 10.685 & $13,6,2,0.05,1$ & 0.97414 & 12.505 \\
\hline & 6 & $6,1,2,0.05,1$ & 0.96033 & 5.721 & $8,3,2,0.05,1$ & 0.97652 & 7.703 \\
\hline & 8 & $\uparrow$ & 0.98119 & $\uparrow$ & $6,2,2,0.10,1$ & 0.96657 & 5.829 \\
\hline & 10 & $4,0,2,0.05,1$ & 0.95168 & 3.929 & $5,1,2,0.05,1$ & 0.96441 & 4.944 \\
\hline & 12 & $\uparrow$ & 0.96911 & $\uparrow$ & $4,1,2,0.15,1$ & 0.96152 & 3.889 \\
\hline \multirow{6}{*}{0.05} & 2 & * & * & * & * & $*$ & * \\
\hline & 4 & $14,4,2,0.05,1$ & 0.95484 & 13.835 & $14,6,2,0.05,1$ & 0.95495 & 13.876 \\
\hline & 6 & $9,2,2,0.10,1$ & 0.95062 & 8.913 & $9,3,2,0.05,1$ & 0.95767 & 8.942 \\
\hline & 8 & $7,1,2,0.05,1$ & 0.97180 & 6.922 & $7,2,2,0.05,1$ & 0.96959 & 6.937 \\
\hline & 10 & $4,0,2,0.05,1$ & 0.95168 & 3.929 & $5,1,2,0.05,1$ & 0.96441 & 4.944 \\
\hline & 12 & $\uparrow$ & 0.96911 & $\uparrow$ & $\uparrow$ & 0.97670 & $\uparrow$ \\
\hline \multirow{6}{*}{0.01} & 2 & * & * & * & * & * & * \\
\hline & 4 & $23,7,2,0.05,1$ & 0.96211 & 22.979 & $22,10,2,0.05,1$ & 0.96865 & 21.971 \\
\hline & 6 & $14,3,2,0.05,1$ & 0.96351 & 13.990 & $14,5,2,0.05,1$ & 0.96350 & 13.993 \\
\hline & 8 & $11,2,2,0.05,1$ & 0.97936 & 10.985 & $10,3,2,0.05,1$ & 0.96969 & 9.989 \\
\hline & 10 & $9,1,2,0.05,1$ & 0.97157 & 8.995 & $8,2,2,0.05,1$ & 0.97196 & 7.990 \\
\hline & 12 & $\uparrow$ & 0.98409 & $\uparrow$ & $6,1,2,0.05,1$ & 0.96033 & 5.992 \\
\hline
\end{tabular}

*: Plan doesn't exist; $\uparrow:$ Use the plan above. 


\section{HUSSAIN ET AL.}

Table 8. Optimal parameters of SkSP-R Plan with $m=2$ under the BS Distribution with shape parameter $\delta=3$

\begin{tabular}{|c|c|c|c|c|c|c|c|}
\hline \multirow{2}{*}{$\beta$} & \multirow{2}{*}{$\mu / \mu_{0}$} & \multicolumn{3}{|c|}{$a=0.5$} & \multicolumn{3}{|c|}{$a=1.0$} \\
\hline & & $n, c, i, f, k$ & $P_{a}\left(p_{1}\right)$ & $\operatorname{ASN}\left(p_{2}\right)$ & $n, c, i, f, k$ & $P_{a}\left(p_{1}\right)$ & $\operatorname{ASN}\left(p_{2}\right)$ \\
\hline \multirow{6}{*}{0.25} & 2 & * & * & * & * & * & * \\
\hline & 4 & $16,7,2,0.05,1$ & 0.95343 & 14.153 & $13,7,2,0.05,1$ & 0.96141 & 11.029 \\
\hline & 6 & $12,5,2,0.05,1$ & 0.97626 & 10.045 & $10,5,2,0.05,1$ & 0.97216 & 8.598 \\
\hline & 8 & $8,0,3,0.10,1$ & 0.95839 & 7.045 & $7,3,3,0.05,1$ & 0.96431 & 6.209 \\
\hline & 10 & $7,2,2,0.05,1$ & 0.96159 & 6.485 & $\uparrow$ & 0.97515 & $\uparrow$ \\
\hline & 12 & $6,2,2,0.15,1$ & 0.95548 & 5.449 & $5,2,2,0.10,1$ & 0.95716 & 4.451 \\
\hline \multirow{6}{*}{0.1} & 2 & * & * & * & * & * & * \\
\hline & 4 & * & * & * & $17,9,2,0.05,1$ & 0.95001 & 16.191 \\
\hline & 6 & $17,7,2,0.05,1$ & 0.97307 & 16.266 & $14,7,2,0.05,1$ & 0.97000 & 13.415 \\
\hline & 8 & $12,4,2,0.05,1$ & 0.95538 & 11.775 & $11,5,2,0.05,1$ & 0.96909 & 10.641 \\
\hline & 10 & $9,3,2,0.10,1$ & 0.95352 & 8.685 & $9,4,2,0.10,1$ & 0.95697 & 8.732 \\
\hline & 12 & $8,2,2,0.05,1$ & 0.95337 & 7.862 & $6,2,2,0.05,1$ & 0.95121 & 5.788 \\
\hline \multirow{6}{*}{0.05} & 2 & * & * & * & * & * & * \\
\hline & 4 & * & * & * & * & $*$ & * \\
\hline & 6 & $18,7,2,0.05,1$ & 0.95821 & 17.738 & $18,9,2,0.05,1$ & 0.96869 & 17.778 \\
\hline & 8 & $12,4,2,0.05,1$ & 0.95537 & 11.775 & $13,6,2,0.05,1$ & 0.97093 & 12.771 \\
\hline & 10 & $10,3,2,0.05,1$ & 0.96005 & 9.812 & $10,4,2,0.05,1$ & 0.95853 & 9.887 \\
\hline & 12 & $8,2,2,0.05,1$ & 0.95337 & 7.862 & $\uparrow$ & 0.97131 & $\uparrow$ \\
\hline \multirow{6}{*}{0.01} & 2 & * & * & * & * & * & * \\
\hline & 4 & * & * & * & * & * & * \\
\hline & 6 & * & * & * & * & * & * \\
\hline & 8 & $20,7,2,0.05,1$ & 0.95893 & 19.973 & $18,8,2,0.05,1$ & 0.95735 & 17.980 \\
\hline & 10 & $16,5,2,0.05,1$ & 0.95892 & 15.979 & $17,7,2,0.05,1$ & 0.95675 & 16.994 \\
\hline & 12 & $14,4,2,0.05,1$ & 0.96214 & 13.983 & $13,5,2,0.05,1$ & 0.96019 & 12.986 \\
\hline
\end{tabular}

*: Plan doesn't exist; $\uparrow:$ Use the plan above.

\section{Advantages of Proposed Plan}

\section{Comparison of SkSP-R Plan under Various Distributions}

In order to compare the performance of the proposed plan, consider Table 9, which gives the ASN values of SkSP-R plan for three distributions namely, Weibull distribution (with shape parameter 1), BS distribution (with shape parameter 1) and EW distribution (with shape parameters 1 and 0.5 ) and for some selected mean life 


\section{SKSP-R PLAN FOR POPULAR STATISTICAL DISTRIBUTIONS}

ratios. The values reported in this table are calculated for $\beta=0.25$ and $a=0.5$ and placed. From this table, observe for the same values of all the parameters, the BS distribution provides the smaller ASN values as compared to the other two distributions. For example, when quality ratio is 4, the ASN of the proposed plan under BS distribution is 3.578. The same obtained under Weibull and EW distributions are 7.153 and 11.858 respectively. This shows the ASN of the proposed plan determined under BS distribution is nearly two times and three times respectively smaller than the ASN of the proposed plan obtained under Weibull and EW distributions. It may be concluded the proposed SkSP-R plan under BS distribution will be admirable in reducing the cost as well as the time of life testing experiments rather than other two distributions.

Table 9. ASN values of proposed plan under various distributions when $\beta=0.25$ and $a=0.5$

\begin{tabular}{|c|c|c|c|}
\hline \multirow{2}{*}{$\mu / \mu_{0}$} & Weibull Distribution & EW Distribution & BS Distribution \\
\hline & $w=1$ & $w=1.0, y=0.5$ & $\delta=1$ \\
\hline 2 & 12.607 & $*$ & 7.965 \\
\hline 4 & 7.153 & 11.858 & 3.578 \\
\hline 6 & 4.426 & 6.156 & 2.943 \\
\hline 8 & 3.613 & $\uparrow$ & $\uparrow$ \\
\hline 10 & $\uparrow$ & 5.384 & $\uparrow$ \\
\hline 12 & $\uparrow$ & $\uparrow$ & $\uparrow$ \\
\hline
\end{tabular}

*: Plan doesn't exist; $\uparrow$ : Use the plan above.

\section{Advantages of SkSP-R Plan over Existing Sampling Plans}

The efficiency of the proposed plan is compared with other existing plans SkSP-2 and SSP. For this comparison, determine the ASN of these three sampling plans under EW with shape parameters $w=1.0$ and $\gamma=0.5$. The ASNs are determined for five values of mean ratios such as $\mu / \mu_{0}=4,6,8,10$ and 12 and also ASNs are presented in Table 10. The ASN of proposed plan is always small when compared to the ASN of other two plans. For example, when $\mu / \mu_{0}=10$ and $\beta=0.01$, the ASN of proposed plan is 14.986, but the ASN of SkSP-2 plan is 17.980 and the ASN of SSP is 35. That is, the ASN of proposed plan is almost two times smaller than the sample size of SSP. Therefore, conclude the decision on the submitted lot will be made with minimum sample size and ASN by using proposed plan rather than SkSP-2 plan and SSP. 
HUSSAIN ET AL.

Table 10. ASN of proposed plan, SkSP-2 and SSP when $w=1$ and $y=0.5$

\begin{tabular}{|c|c|c|c|c|}
\hline$\beta$ & $\mu / \mu_{0}$ & $S k S P-R$ & SkSP-2 & SSP \\
\hline \multirow{5}{*}{0.25} & 4 & 11.858 & 12.937 & 24 \\
\hline & 6 & 6.156 & 8.944 & 17 \\
\hline & 8 & 6.156 & 6.165 & 15 \\
\hline & 10 & 5.384 & 6.165 & 13 \\
\hline & 12 & 5.384 & 5.402 & 10 \\
\hline \multirow{5}{*}{0.1} & 4 & 16.440 & 19.469 & * \\
\hline & 6 & 10.502 & 10.504 & 25 \\
\hline & 8 & 7.633 & 10.504 & 20 \\
\hline & 10 & 7.633 & 7.634 & 20 \\
\hline & 12 & 7.633 & 7.634 & 18 \\
\hline \multirow{5}{*}{0.05} & 4 & 20.739 & 23.756 & * \\
\hline & 6 & 11.784 & 14.746 & * \\
\hline & 8 & 11.784 & 11.784 & 28 \\
\hline & 10 & 8.853 & 11.784 & 22 \\
\hline & 12 & 8.853 & 8.854 & 22 \\
\hline \multirow{5}{*}{0.01} & 4 & 32.976 & 34.956 & * \\
\hline & 6 & 20.976 & 23.974 & * \\
\hline & 8 & 17.980 & 17.980 & * \\
\hline & 10 & 14.986 & 17.980 & 35 \\
\hline & 12 & 14.986 & 14.986 & 29 \\
\hline
\end{tabular}

*: Plan doesn't exist

\section{Applications}

Example: Suppose that a manufacturer adopts the proposed plan when the shape parameter is unknown. The specified life of the product is $\mu_{0}=1000 \mathrm{~h}$ and the test duration is $500 h$. Assuming that $\alpha=0.05, \beta=0.10, a=0.5$ and $\mu / \mu_{0}=2$. It is known that from the past history, the lifetime of the product follows the Weibull distribution. In order to estimate the shape parameter $w$, failure data were collected from 10 products of the previous lots as follows: 507, 720, 892, 949, 1031, 1175, $1206,1428,1538,2083$ respectively. This data follows a Weibull distribution. The distribution fit was also tested by goodness of fit and its P-P plot is shown in Figure 1. Then, the maximum likelihood estimate (MLE) of the shape parameter is obtained by $\hat{w}=2.8229$. So, let us assume that $\mathrm{w}=3$ now. From Table 3 the optimal design parameters are obtained as $n=31, c=0, i=2, f=0.10$ and $k=1$. 


\section{SKSP-R PLAN FOR POPULAR STATISTICAL DISTRIBUTIONS}

Thus, the sampling plan can be operated as follows: select a sample of $n=31$ items from the lot of the product. Conduct the life test for the specified duration of 500 hours and count number of sample items failed before the testing time is reached. Accept the lot if none of the sample items failed before the experiment time. If 2 consecutive lots have been accepted under normal inspection, then switch to the skipping inspection. Inspect a fraction of (1/10) items and continue skipping inspection until a lot is rejected. If a lot is rejected after 1 sampled lot has been accepted, use the resampling procedure.

During the resampling procedure, inspect the non-acceptance lot 2 times (because $m=2$ ) using the reference plan. Accept the lot under resampling procedure if it has been accepted on the first resubmission and continue skipping inspection. Otherwise, reject the lot and continue revert to normal inspection.

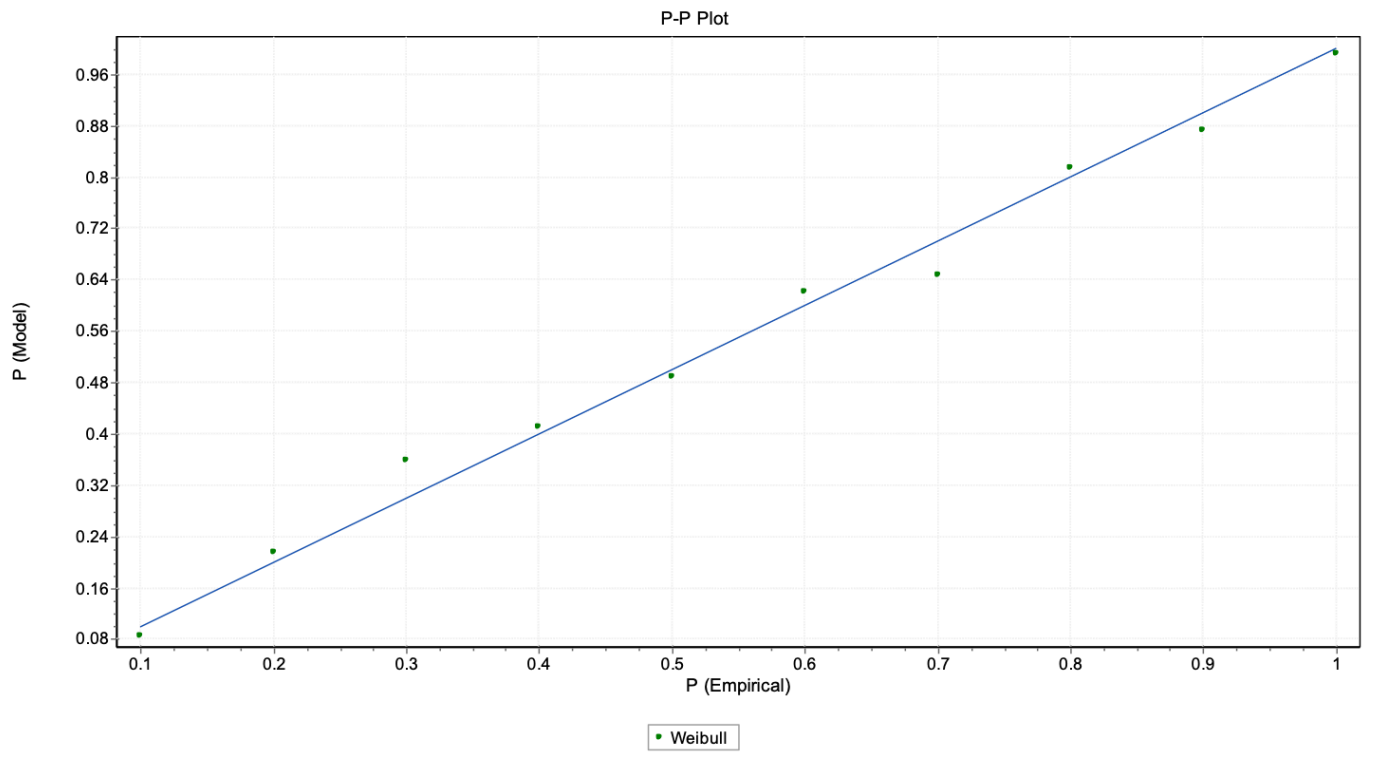

Figure 1. PP Plot for the data given in example

\section{Conclusion}

The design of the SkSP-R sampling plan was presented for three widely used distributions in life testing area. Tables were developed for the selection of optimal parameters of proposed plan under these three distributions for the industrial implementation. A comparison was given between the ASN of the proposed plan 


\section{HUSSAIN ET AL.}

obtained under three distributions and it has been found that BS distribution has economical advantages over the other two distributions. The efficiency of the SkSP-R plan was compared over the three other existing sampling plans. The proposed plan is better one for application in the industries for the inspection/testing of electric products. The proposed sampling plan can be extended for some other distributions as a future research.

\section{Acknowledgements}

The authors are thankful to the editor and the anonymous reviewers for their constructive comments which greatly improved the presentation of the paper.

\section{References}

Aslam, M., Balamurali, S., Jun, C.-H. \& Ahmad, M. (2013a). Optimal Design of Skip Lot Group Acceptance Sampling Plans for the Weibull Distribution and the Generalized Exponential Distribution. Quality Engineering, 25(3), 237-246.

https://doi.org/10.1080/08982112.2013.769053

Aslam, M., Balamurali, S., Azam, M. \& Jun, C.-H. (2014a). Skip-Lot Sampling Plan of Type SkSP-2 with Two-Stage Group Acceptance Sampling Plan as Reference Plan. Communications in Statistics - Simulation and Computation, 43(4), 777-789. https://doi.org/10.1080/03610918.2012.715224

Aslam, M., Balamurali, S., Jun, C.-H. \& Ahmad, M. (2010). Optimal Designing of a Skip-lot Sampling Plan by Two Point Method. Pakistan Journal of Statistics, 26(4), 585-592.

Aslam, M., Balamurali, S., Jun, C-H. \& Ahmad, M. (2012a). An Optimal Design of a Skip-Lot Sampling Plan of Type V by Minimizing Average Sample Number. Pakistan Journal of Statistics, 28(1), 131-140.

Aslam, M., Balamurali, S., Jun, C-H. \& Ahmad, M. (2012b). Optimal Designing of an SkSP-V Skip-lot Sampling Plan with Double Sampling Plan as the Reference Plan. International Journal of Advanced Manufacturing Technology, 60(5-8), 733-740. https://doi.org/10.1007/s00170-011-3635-5

Aslam, M., Balamurali, S., Jun, C-H. \& Rasool, M. (2013b). Optimal Designing of Skip-lot Sampling Plan of Type SkSP-2with Group Acceptance Sampling Plan as the Reference Plan under Burr Type XII Distribution. Journal of Statistical Computation and Simulation, 83(1), 37-51. https://doi.org/10.1080/00949655.2011.600697 


\section{SKSP-R PLAN FOR POPULAR STATISTICAL DISTRIBUTIONS}

Aslam, M., Srinivasa Rao, G., Khan, N. \& Jun, C.-H. (2014b). SkSP-V Sampling Plan for the Exponentiated Weibull Distribution. Journal of Testing and Evaluation, 42(3), 687-694. https://doi.org/10.1520/jte20130051

Balamurali, S. \& Jun, C.-H. (2011). A New System of Skip-lot Sampling Plans Having a Provision for Reducing Normal Inspection. Applied Stochastic Models in Business and Industry, 27(3), 348-363. https://doi.org/10.1002/asmb.844

Balamurali, S., Jun, C.-H. \& Collani, von E. (2008). Skip-lot Sampling and Chain Sampling. In F. Ruggeri et al. (Eds.) Encyclopedia of Statistics in Quality and Reliability. NY: Wiley. https://doi.org/10.1002/9780470061572.eqr142

Balamurali, S. \& Subramani, J. (2012). Optimal Designing of Skip-lot Sampling Plan of Type SkSP-2 with Double Sampling Plan as the Reference Plan. Journal of Statistical Theory and Practice, 6(2), 354-362. https://doi.org/10.1080/15598608.2012.673897

Balamurali, S. \& Subramani, J. (2018). Optimal Designing of Skip-lot Sampling

Plan for the Inspection of Normally Distributed Quality Characteristics. Transactions of the Institute of Measurement and Control, 40(7), 2240-2248.

https://doi.org/10.1177/0142331217700466

Balamurali, S., Aslam, M. \& Jun, C.-H. (2014a). A New System of Product Inspection Based on Skip-Lot Sampling Plans Including Resampling. The Scientific World Journal, 192412, 1-6, https://doi.org/10.1155/2014/192412

Balamurali, S., Aslam, M. \& Jun, C-H. (2014b). Economic Design of SkSP-R Skip-lot Sampling Plan. Journal of Testing and Evaluation, 43(5), 1205-1210. https://doi.org/10.1520/jte20140081

Cao, Y. \& Subramaniam, V. (2013). Improving the Performance of Manufacturing Systems with Continuous Sampling Plans. IIE Transactions, 45(6), 575-590. https://doi.org/10.1080/0740817x.2012.733485

Carr, W. E. (1982). Sampling Plan Adjustment for Inspection Error and Skip-lot Plan. Journal of Quality Technology, 14(3), 10-18. https://doi.org/10.1080/00224065.1982.11978802

Cox, D. C. (1980). Skip-lot Sampling Plans. Quality, 21(8), 26-27.

Dodge, H. F. (1955). Skip-lot Sampling Plan. Industrial Quality Control, 11(5), 3 5. https://doi.org/10.1080/00224065.1977.11980786

Duffuaa, S. O., Turki, U. M. \& Kolus, A. A. (2009). Process-targeting Model for a Product with Two Dependent Quality Characteristics Using Acceptance Sampling Plans. International Journal of Production Research, 47(14), 4031-4046. https://doi.org/10.1080/00207540701644243 


\section{HUSSAIN ET AL.}

Govindaraju, K. and Ganesalingam, S. (1997). Sampling Inspection for Resubmitted Lots. Communications in Statistics - Simulation and Computation, 26(3), 1163-1176. https://doi.org/10.1080/03610919708813433

Hsu, J. I. S. (1980). A Cost Model for Skip-lot Destructive Testing. IEEE Transaction on Reliability, R-26(1), 70-72. https://doi.org/10.1109/tr.1977.5215081

Hussain, J., Aslam, M. \& Jun, C.-H. (2014). Inspection of Batches Through Skip-R Lot Sampling Plan. Journal of Testing and Evaluation, 42(2), 437-443. https://doi.org/10.1520/jte20130100

Lemonte, A. J., Cribari-Neto, F. \& Vasconcellos, K. L. P. (2007). Improved Statistical Inference for the Two-Parameter Birnbaum-Saunders Distribution.

Computational Statistics and Data Analysis, 51(9), 4656-4681.

https://doi.org/10.1016/j.csda.2006.08.016

Liebesman, B. S. (1987). The Development of an Attribute Skip-lot Sampling Standard. In H. J. Lenz et al. (Eds). Frontiers in Statistical Quality Control, 3, 3-33. https://doi.org/10.1007/978-3-662-11787-3_1

Mudholkar, G.S. \& Srivastava, D.K. (1993). Exponentiated Weibull family for Analyzing Bathtub Failure-Real Data. IEEE Transactions on Reliability, 42(2), 299-302. https://doi.org/10.1109/24.229504

Parker, R. D. \& Kessler, L. (1981). A Modified Skip-lot Sampling Plan. Journal of Quality Technology, 13(1), 31-35. https://doi.org/10.1080/00224065.1981.11980983

Perry, R. L. (1970). A System of Skip-lot Sampling Plans for Lot Inspection. Ph.D. Thesis, Rutgers-The State University, New Brunswick, New Jersey.

Perry, R. L. (1973). Skip-lot Sampling Plans. Journal of Quality Technology, 5(3), 123-130. https://doi.org/10.1080/00224065.1973.11980585

Taylor, W. A. (1997). Selecting Statistically Valid Sampling Plans. Quality Engineering, 10(2), 365-370. https://doi.org/10.1080/08982119708919144 